\title{
Fuzzy logic enhancement of material hardening parameters obtained from tension-compression test
}

Received: 20 March 2019 / Accepted: 10 June 2019 / Published online: 17 June 2019

(C) The Author(s) 2019

\begin{abstract}
This paper presents the determination of material hardening parameters with the use of the fuzzy set theory. The hardening parameters were initially predicted from measurements taken in the cyclic tensioncompression test. The experimental hysteresis loop was compared to numerical one obtained by the integration of the plastic flow rule. The nonlinear combined hardening model-Voce isotropic hardening and FrederickArmstrong kinematic hardening - was considered here. After the initial selection, the hardening parameters were adjusted in the optimization problem using the least-squares method. An approximation error of the hysteresis loop was minimized. Finally, nonlinear isotropic and kinematic hardening parameters were assumed to be fuzzy variables. Hardening parameters obtained in the optimization problem were randomly scattered up to $20 \%$, and the membership functions associated with them were computed. The approximation error of the hysteresis loop was found for each selection of the hardening parameters providing the output membership function associated with this error. The $\alpha$-level optimization method was used as the main numerical tool, while the extension principle was tested only as the reference solution. In the defuzzification process, the most reliable hardening parameters were found.
\end{abstract}

Keywords Fuzzy logic $\cdot \alpha$-Level optimization $\cdot$ Frederick-Armstrong kinematic hardening model $\cdot$ Cyclic tension-compression test $\cdot$ Mechanical engineering

\section{Introduction}

The structural integrity of engineering structures under cyclic loading is an important aspect for different industries. Under cyclic loading conditions, materials experience repeated tensile and compressive load showing different behaviors under monotonic loading [1,2]. Many researchers examined the behavior of materials and structures which underwent the cyclic tension-compression test. Kim et al. [3] tested the influence of loading rate on the fracture behavior of nuclear piping materials under cyclic loading conditions. In another work, Kim et al. [4] examined the behavior of CF8A austenite cast stainless steel in a tension-compression test. The behavior of materials under cyclic loading conditions is an issue which might be considered both in microand macroscale.

Most of metals, which undergo the plastic deformation, have ability to strain hardening. The hardening is defined as an increase in the yield stress caused by an increase in the plastic deformation [5]. The hardening

Communicated by Francesco dell'Isola.

M. Wójcik $(\bowtie) \cdot$ A. Skrzat

Department of Materials Forming and Processing, Faculty of Mechanical Engineering and Aeronautics,

Rzeszow University of Technology, 8 Powstańców Warszawy Ave., 35-959 Rzeszow, Poland

E-mail: m.wojcik@prz.edu.pl

A. Skrzat

E-mail: askrzat@prz.edu.pl 
effect results from the dependence between the yield stress and the history of the plastic straining [6]. In numerical simulations of the material hardening behavior, different hardening models might be used. The isotropic models assume a uniform expansion of the yield surface without its translation in the stress space. The translation of the yield surface without the change in its size is described by kinematic hardening models. The Frederick-Armstrong (F-A) model [7] is a commonly used nonlinear kinematic hardening model which includes the Bauschinger effect. In the Chaboche kinematic hardening model, the back stress is the superposition of several kinematic laws [8]. The Chaboche model enables a better description of the material response to the external load than F-A model and is preferred in simulations of cyclic load problems, e.g., shakedown or ratcheting [6]. Other popular kinematic hardening models are Ohno-Wang, Teodosiu-H and Yoshida-Uemori [9]. The combination of nonlinear kinematic and isotropic hardening laws was included in Chaboche and Lemaitre model [10].

The determination of hardening parameters and modeling of hardening curves are very important problems under scientific consideration. In [11], the usefulness of the finite element model updated procedure (FEMU) for the identification of hardening parameters of the 2024-T3 aluminum alloy was examined. The determination of hardening parameters by means of the virtual field's method (VFM) was tested in [12]. The influence of the number of tension-compression cycles on the values of hardening parameters with the use of Chaboche and Lemaitre mixed hardening model was included in [6].

The determination of material parameters can be also based on the micro-macro identifications. Placidi et al. [13] have identified the set of parameters which characterized the model of homogeneous linear second gradient D4 orthotropic continuum. In this research, the set of constitutive parameters includes: the material of fibers, fibers cross sections and the internal rotational springs positioned at each intersection between the two groups of fibers. Misra and Poorsolhjouy [14] have used the continuum model of the granular media enriched by non-classical terms in order to describe the material behavior more precisely. The higher-order elastic constants of the enhanced continuum model were determined from discrete simulations subjected to relevant boundary conditions. After that, the elastic constants were used for the determination of the microscale stiffness coefficient of the continuum model. In [15], the Dahl and LuGre considered models which describe the friction in the concrete specimens subjected to uniaxial compressive loading. De Angelo et al. [16] showed the usefulness of homogenized models for the description of the pantographic microstructure of metallic specimens. Detailed information concerning the aforementioned research is included in [13-16].

The proper selection of hardening parameters plays an important role both in experimental and numerical calculations. However, material hardening parameters obtained from experimental investigations and/or from numerical simulations can be found with a certain precision. Several samples made from the same material can provide little different hardening parameters. Nowadays, soft-computing methods, e.g., neural networks and genetic algorithms, become popular approaches which allow improving the magnitudes of material hardening data. In this paper, a general approach to enhance material parameters by means of the fuzzy set theory is proposed.

The advantage of the fuzzy set theory is that it can be applied in the determination of hardening parameters assuming their scattering. The fuzzy logic is a very efficient non-deterministic computational method for solving engineering problems for which the knowledge about considered model is incomplete or even unknown. The fuzzy theory and fuzzy sets are described in [17]. In [18], the application of the fuzzy logic for solving simple mechanical problems was presented. The response of the Bodner-Partom material to external loads with the use of fuzzy logic was investigated in [19]. The application of the fuzzy set theory for the determination of material strain hardening parameters in Hollomon equation based on the Heyer's test was described in [20]. In [21], the fuzzy logic model was also used for the prediction of failure of composite plates under different temperatures. Akkurt et al. [22] applied the fuzzy logic model for the prediction of the compressive strength of cement mortar. The fuzzy set theory has been also applied in commercial applications. Vygnanov and Fironov [23] proposed the fuzzy logic system estimating the risks of the high-speed railway. Guclu and Metin [24] tested the fuzzy logic system to control the light rail transport vehicles vibrations. The application of the fuzzy logic for the durability assessment of bridge structures is presented in [25].

In this paper, the application of the fuzzy set theory for adjusting material hardening parameters found from experimental stress-strain curves obtained in the cyclic tension-compression tests is presented. The $\alpha$-level optimization method was used as the main numerical tool. Material hardening parameters are assumed to be fuzzy variables. Numerical hysteresis loops are compared to experimental ones, and the fuzzed error of approximation was found. In the defuzzification process, the most reliable hardening parameters are found. The advantage of using the fuzzy set theory over typical statistical methods is that the fuzzy logic considers not only the scattering of input and output parameters but also the usually nonlinear properties of the mapping 
model (the plastic flow rule). The future goal of this research is the analysis of sophisticated elastic-plastic material models like generalized elasticity and plasticity models which includes more nonlinear effects, e.g., creep and relaxation, as well as, visco-plastic models, etc. In the further research concerning the modeling of the hardening curves, the Bodner-Partom model (generalized plasticity) as well as the Chaboche model will be tested.

Before such a study, the classical elastic-plastic nonlinear models are considered, namely Voice isotropic and Frederick-Armstrong kinematic models. It might be treated as a first stage of a research concerning the usefulness of fuzzy logic for the determination of hardening parameters.

\section{Combined hardening: problem formulation}

In the numerical simulation of elastic-plastic stress-strain curves, the Hooke's law and the plastic flow rule associated with von Mises yield criterion were used. For simplicity, the explicit integration was used. The basic equations for combined isotropic-kinematic hardening model are presented below [26].

The yield criterion is described by the following Eq. (1) [27]:

$$
\mathrm{f}(\boldsymbol{\sigma}, \mathbf{x}, p)=\sqrt{\frac{3}{2}\left(\boldsymbol{\sigma}^{\prime}-\mathbf{x}\right):\left(\boldsymbol{\sigma}^{\prime}-\mathbf{x}\right)}-\sigma_{y}-r(p)
$$

where $\sigma$ and $\sigma^{\prime}$ are the stress and deviatoric stress, respectively, $\mathbf{x}$ is the back stress, $\sigma_{y}$ is the yield stress, $r(p)$ is the isotropic hardening function, and $p$ is the effective plastic strain. Elastic deformation occurs if the yield function $\mathrm{f}(\boldsymbol{\sigma}, \mathbf{x}, p)<0$, while the plastic flow takes place for $\mathrm{f}=0[28,29]$.

The effective plastic strain is described by the following formula (2) [30]:

$$
\mathrm{d} p=\sqrt{\frac{2}{3} \mathrm{~d} \boldsymbol{\varepsilon}^{\mathrm{p}}: \mathrm{d} \boldsymbol{\varepsilon}^{\mathrm{p}}}
$$

where $\mathrm{d} \boldsymbol{\varepsilon}^{\mathrm{p}}$ is the increase in the plastic strain.

The consistency condition is described by Eq. 3:

$$
\mathrm{d} f=\frac{\partial f}{\partial \boldsymbol{\sigma}}: \mathrm{d} \boldsymbol{\sigma}+\frac{\partial f}{\partial \mathbf{x}}: d \mathbf{x}+\frac{\partial f}{\partial p} \mathrm{~d} p=0
$$

The normality condition is (4):

$$
\mathrm{d} \boldsymbol{\varepsilon}^{\mathrm{p}}=\mathrm{d} \lambda \frac{\partial f}{\partial \boldsymbol{\sigma}}
$$

where $d \lambda$ is the plastic multiplier which can be found from the consistency condition (3). The plastic flow associated with the von Mises yield criterion is described by Eq. 5 [27]:

$$
\mathrm{d} \boldsymbol{\varepsilon}^{\mathrm{p}}=\mathrm{d} \lambda \frac{3 \sigma^{\prime}}{2 \sigma_{e}}
$$

where $\sigma_{e}$ is the effective stress. Otherwise, $\mathrm{d} \boldsymbol{\varepsilon}^{\mathrm{p}}$ equals 0 [28].

The back stress increment $d \mathbf{x}$ in Frederick-Armstrong nonlinear kinematic hardening model [31] is:

$$
\mathrm{d} \mathbf{x}=\frac{2}{3} c \mathrm{~d} \boldsymbol{\varepsilon}^{\mathbf{p}}-\gamma \mathbf{x} \mathrm{d} p
$$

where $c$ is the material constant, $\gamma$ defines the rate of the kinematic hardening saturation, and $\mathrm{d} p$ is the equivalent plastic strain increment.

The increment in isotropic hardening $d r(p)$ is determined by Voce isotropic law [32] as:

$$
\mathrm{d} r(p)=b(Q-r) \mathrm{d} p
$$

where $Q$ is the saturated value of $r$ and $b$ determines the rate at which the saturation is achieved.

Assuming the superposition of elastic and plastic strain increments $d \boldsymbol{\varepsilon}=d \boldsymbol{\varepsilon}^{\mathrm{e}}+d \boldsymbol{\varepsilon}^{\mathrm{p}}$, the Hooke's law is described by the following Eq. (8):

$$
\mathrm{d} \sigma=\mathbf{C}\left(d \boldsymbol{\varepsilon}-\mathrm{d} \boldsymbol{\varepsilon}^{\mathrm{p}}\right)
$$


In (8), the Voigt notation is used for stress and strain tensors and $\mathbf{C}$ is the constitutive matrix. Combining Eqs. (1-8), one can express the plastic multiplier in terms of the strain increment:

$$
\mathrm{d} \lambda=\frac{\mathbf{n} \cdot \mathbf{C d} \boldsymbol{\varepsilon}}{\mathbf{n} \cdot \mathbf{C n}-\gamma \mathbf{n} \cdot \mathbf{x}+\frac{2}{3} \mathbf{c n} \cdot \mathbf{n}+b(Q-r)}
$$

where $\mathbf{n}=\frac{\partial f}{\partial \sigma}$. It should be mentioned that for von Mises yield criterion, $\mathrm{d} \lambda=\mathrm{d} p$.

The plastic multiplier can be also expressed in terms of the stress increment. After substituting (8) into (9) and some transformations, the plastic multiplier is described by Eq. 10.

$$
\mathrm{d} \lambda=\frac{\mathbf{n} \cdot \mathrm{d} \boldsymbol{\sigma}}{\frac{2}{3} c \mathbf{n} \cdot \mathbf{n}-\gamma n \cdot \mathbf{x}+b(Q-r)}
$$

Equations (1) to (8) were used in developing of numerical algorithm for an elastic-plastic material response to the kinematic load. For assumed explicit integration, the algorithm executes as follows:

1. For a given strain increment, check the yield condition (1)

2. If $f<0$, find the stress increment from (8)

3. If $f=0$,

- find the plastic multiplier from (9)

- find the plastic strain increment from (5), the back stress increment from (6) and the isotropic hardening increment from (7)

- find the stress increment from (8)

- upgrade strain, stress, back stress and isotropic hardening at the end of this step.

For a simple uniaxial loading, the explicit form of the stress increment vs. the strain increment for $f=0$ can be derived (11):

$$
\mathrm{d} \sigma=E\left(1-\frac{E}{E+c-\gamma \cdot x+b(Q-r)}\right) \mathrm{d} \varepsilon
$$

where $E$ is the Young's modulus.

\section{General description of the fuzzy logic analysis}

Fuzzy logic allows including various uncertainty of the input and output data [33] in an analysis. In general, it represents a mapping of fuzzy input variables into the result space. The determination of the fuzzy output variable includes some steps among which the most important are the fuzzification and defuzzification (Fig. 1). In the fuzzification step, the membership functions associated with input variables should be defined. After that, the fuzzy output variables are determined with the application of the mapping model [34]. The mapping model might be determined explicitly by the function, can be described by means of linguistic variables or can be a sophisticated algorithm, e.g., the system of differential equations.

A number of fuzzy logic methods can be applied in order to obtain the membership functions for fuzzy output variables. In this research, the $\alpha$-level optimization as well as the extension principle method was tested. In the extension principle, the membership function for $z$ output variable is calculated with the use of minimum and supreme operators according to the following formula (12):

$$
\mu(z)=\sup \min [\mu(x), \mu(y)]
$$

where $\mu(x)$ and $\mu(y)$ are membership functions of $x$ and $y$ input variables, $z$ is an output variable, and $\mu(z)$ is the membership function of $z$ output variable.

For randomly selected $x$ and $y$ fuzzy input variables, their membership functions $\mu(x)$ and $\mu(x)$ are computed. After that, the minimum values of $\mu(x)$ and $\mu(x)$ are determined. In this way, $z$ variable can be associated with several $\mu(z)$ values. Thus, the supremum operator is used. For floating-point numbers, the maximum $\mu(z)$ value should be searched for in the $\mathrm{z} \pm \Delta \mathrm{z}$ interval.

The main disadvantage of the extension principle method is the favoring of minimum values of $\mu(z)$. (The minimum operator is applied at first.) Relatively smooth envelope (an application of supremum operator) can be obtained only for a very dense searching for $x$ and $y$ fuzzy input variables. This is time-consuming process. 


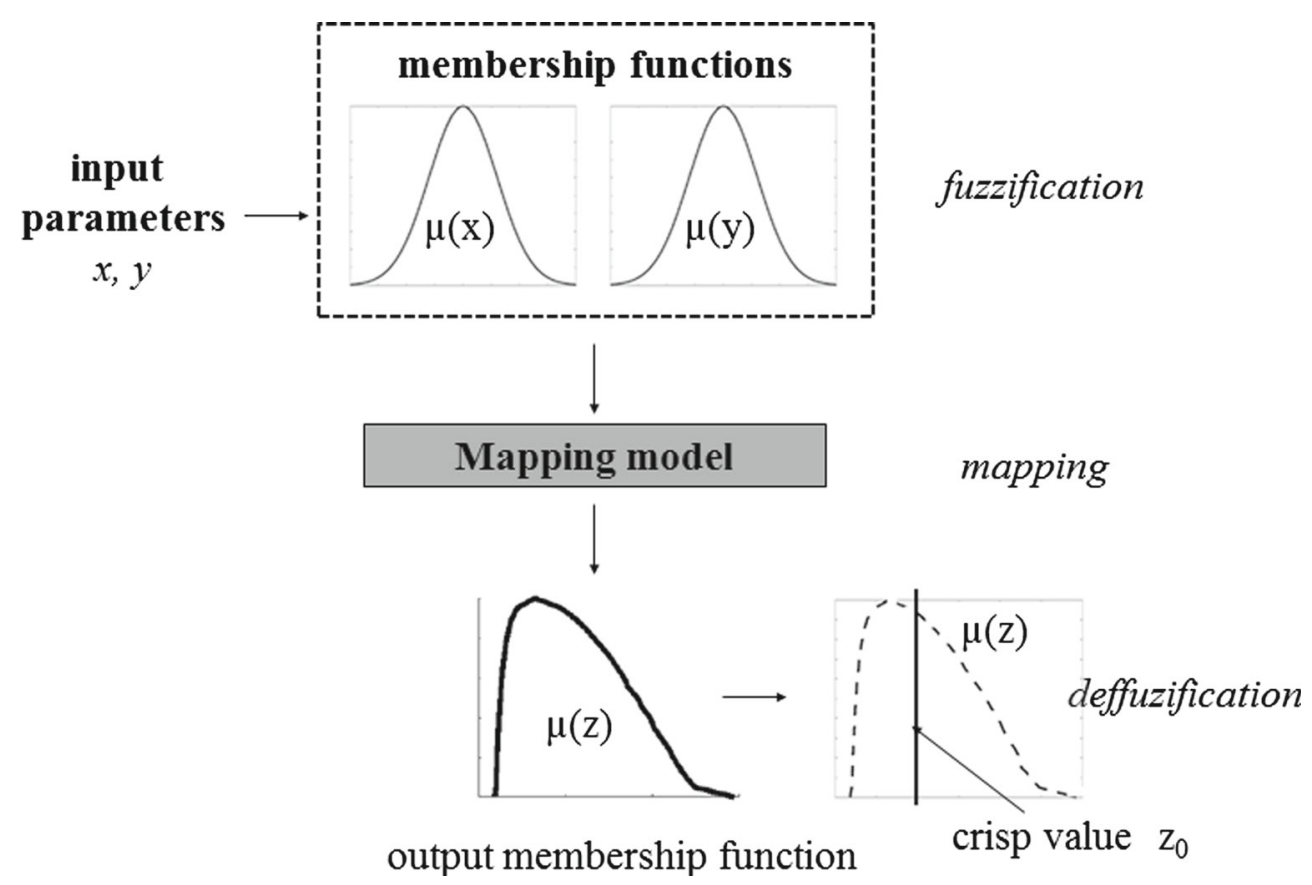

Fig. 1 Steps of the fuzzy analysis
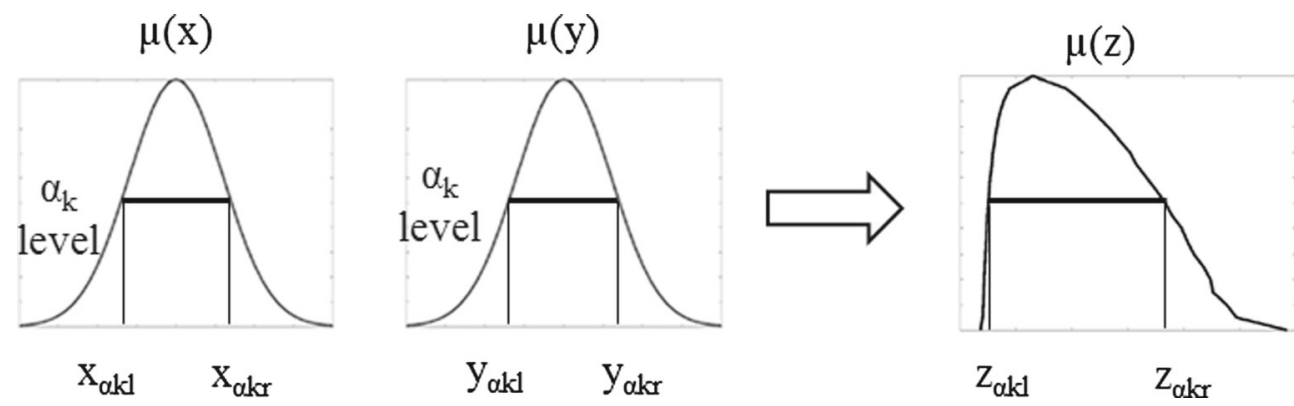

Fig. 2 The $\alpha$-level optimization method

The results obtained by means of the extension principle usually receive a form of a jagged solution. It is worth noting that the final result also depends on the assumed precision $\Delta z$.

If the mapping function requires a lot of calculations, the extension principle is not effective. Much better results might be obtained by the application of $\alpha$-level optimization method [17].

In this approach [18], the membership functions of $x$ and $y$ fuzzy input variables are separated into sufficient number of $\alpha$-levels. For the specific $\alpha_{k}$ level $\alpha_{k}[0 ; 1]$ the boundary values $x_{\alpha k l}, x_{\alpha k r}, y_{\alpha k l}$ and $y_{\alpha k r}$ are computed (Fig. 2). For randomly selected $x \in\left[x_{\propto k l}, x_{\alpha k r}\right]$ and $y \in\left[y_{\propto k l}, y_{\alpha k r}\right]$, the minimum $z_{\alpha k l}$ and maximum $z_{\alpha k r}$ are found. The above procedure is repeated for all $\alpha$-levels. Extreme $z$ values for all $\alpha$-levels determine the shape of the fuzzy output membership function $\mu(z)$.

The $\alpha$-optimization method can be used only if the mapping operator is continuous and the fuzzy result space is convex. In comparison with the extension principle, the $\alpha$-level optimization method enables to obtain a smooth shape of the output membership function with the same number of calculations. For this reason, the $\alpha$-optimization method was used as the main numerical tool in this research. The extension principle was applied only in order to check the consistency of the obtained results.

In the defuzzification procedure, the most valuable result is selected from the fuzzy result space. There are several defuzzification methods, e.g., the height method, the mass center method, level rank method or Jain and Chen method. In this research, the mass center method was applied. This concept is based on the searching for the center of the space below the fuzzy output membership function. Mass center is defined by 


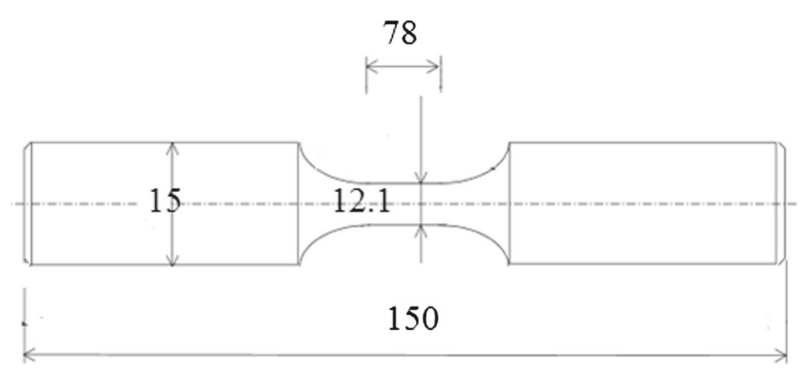

Fig. 3 Dimensions (mm) of the specimen used in this experimental research

the following formula [17]:

$$
z_{0}=\frac{\int z \cdot \mu(z) \mathrm{d} z}{\int \mu(z) \mathrm{d} z}
$$

where $z_{0}$ is the most reliable value of the $z$ variable and $\mu(z)$ is the membership function of $z$ variable.

\section{Experimental investigations}

In the experimental investigations, the hardening curves for samples subjected to cyclic tension-compression loads are recorded. The cyclic tests with certain strain amplitudes enable to identify material hardening parameters [35].

The strain-controlled cyclic tension-compression test was carried out at room temperature on ZWICK/ ROELL Z030 testing machine. The deformation of $\pm 5 \%$ of measuring base was applied. The sample was made of PA6 aluminum alloy. The specimen geometry is shown in Fig. 3.

The experimental curve for PA6 alluminium alloy subjected to the tension-compression test is presented in Fig. 4. Engineering quantities $\sigma_{\text {nom }}$ and $\varepsilon$ registered in the test are transferred into true ones by formulas (14) and (15) assuming material constant volume for the plastic deformation.

$$
\begin{aligned}
& \sigma=\sigma_{\text {nom }}(1+\varepsilon) \\
& \phi=\ln (1+\varepsilon)
\end{aligned}
$$

where $\sigma_{\text {nom }}$ is the engineering (nominal) stress, $\varepsilon$ is the engineering strain, $\sigma$ is the true stress, and $\phi$ is the logarithmic strain.

The true stress in (14) is defined as the force related to the current cross section. The logarithmic strain (15) enables the superposition of strains in the large deformation analysis. A material subjected to finite deformations will have the same stress-strain behavior in tension and compression if the true stress and the logarithmic strain are applied.

\section{Numerical simulations of the hysteresis loops}

The equations presented in Sect. 2 are used in order to develop the numerical program which computes the elastic-plastic material response to the assumed strain increment. This program implemented in MATLAB simulates the cyclic tension-compression test. For simplicity, the explicit integration procedure is applied with about four thousand integration steps for single hysteresis loop. (Explicit procedure is conditionally stable.) For initially selected $b, Q, c, \gamma$ hardening parameters, the stress-strain curve is found as shown in Fig. 5.

One can see from Fig. 5 that quite a good agreement between experiment and numerical simulation was achieved. In the initial selection of hardening parameters, the following indicators are used:

- the size of the initial yield surface $\pm \sigma_{Y 0}$ and the size of the yield surface for the final loop-determination of $Q$ parameter

- a number of loops up to hysteresis stabilization-parameter $b$

- extrapolated shift of the yield stress for the final stable loop-the ratio $\frac{c}{\gamma}$

- curvature between elastic and plastic response-parameter $\gamma$ 


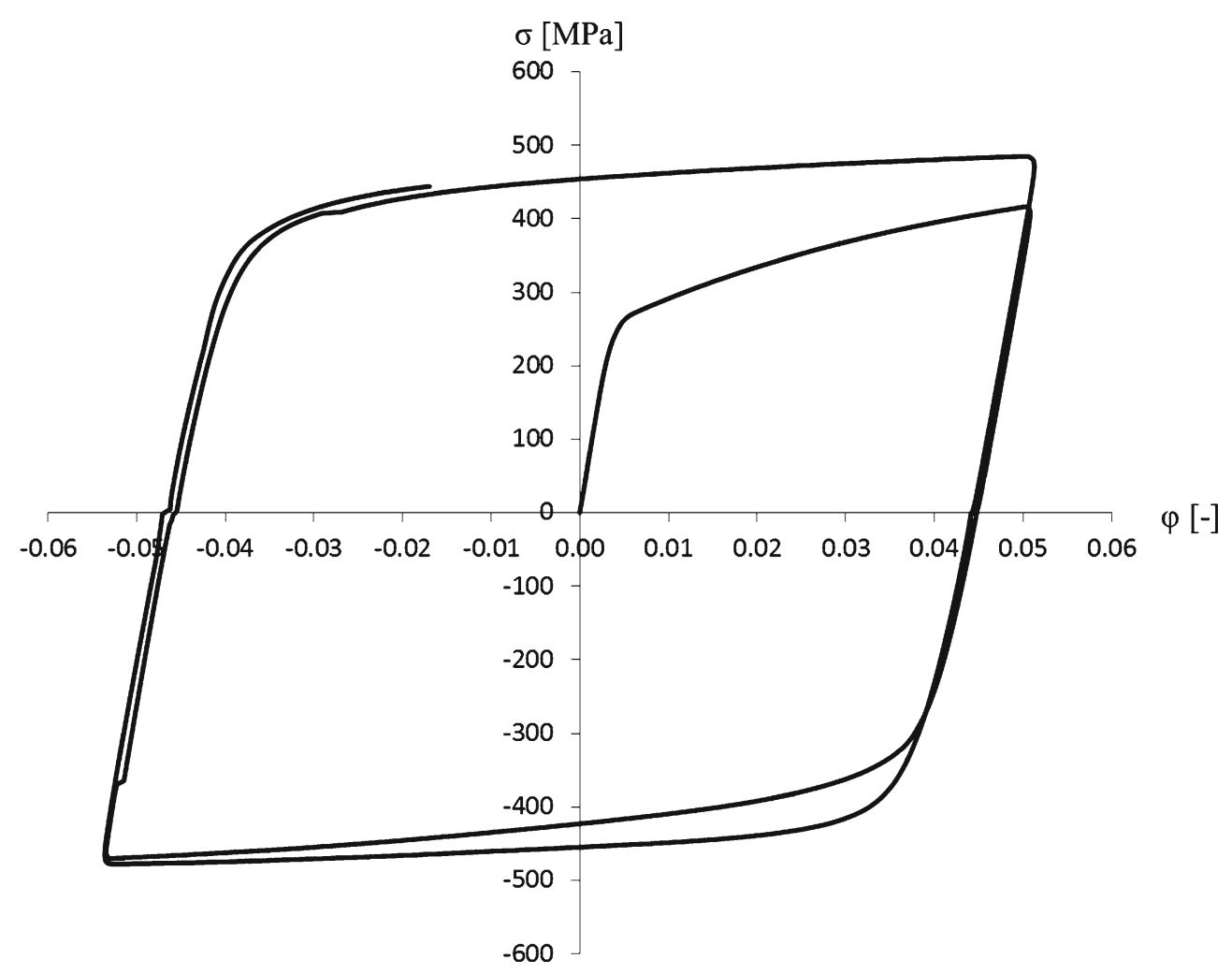

Fig. 4 The experimental curve for PA6 aluminum alloy
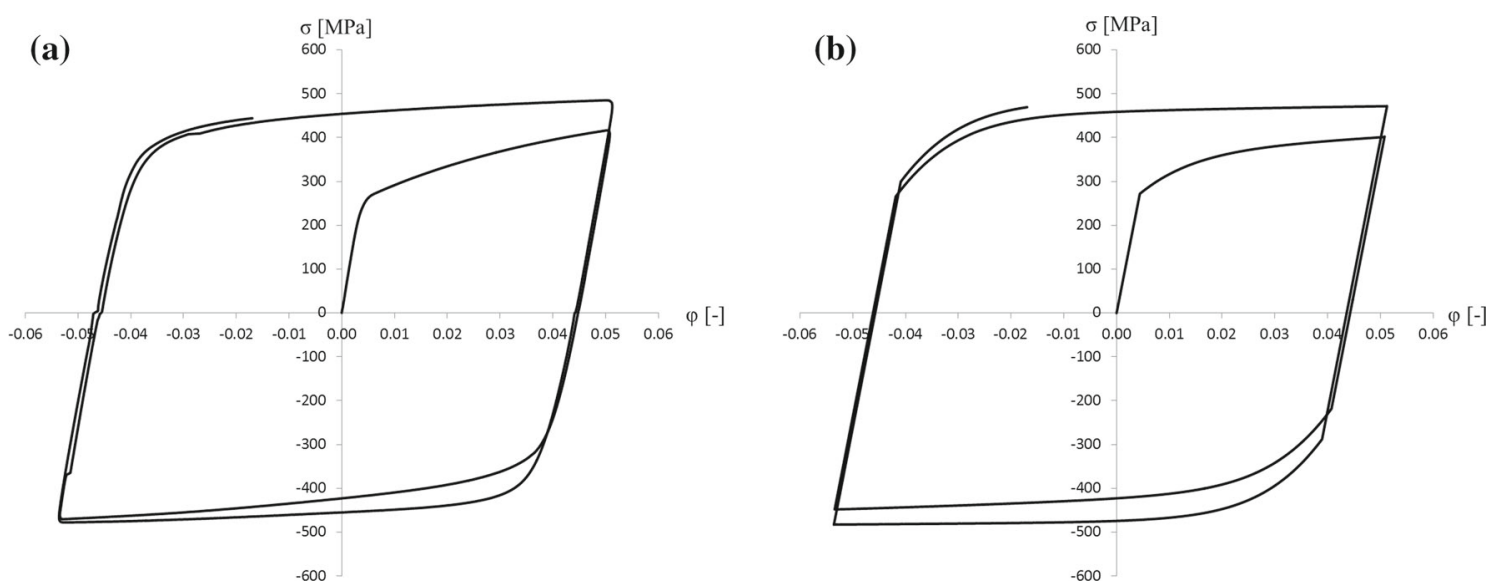

Fig. 5 Comparison of experimental (a) and numerical (b) curves for a cyclic tension-compression test

As the initial guess, the following hardening parameters are selected: $Q=154 \mathrm{MPa}, b=7, c=$ $12,000 \mathrm{MPa}, \gamma=100\left(\frac{c}{\gamma}=120 \mathrm{MPa}\right)$.

Better fitting of numerically simulated curve to the experimental one can be achieved by means of the optimization approach [36]. Here, the following error norm $(B)$ was introduced:

$$
\|B\|=\sqrt{\int_{\varepsilon}\left(\sigma_{\exp }-\sigma_{\text {app }}\right)^{2} \mathrm{~d} \varepsilon}
$$

where $\sigma_{\text {exp }}$ and $\sigma_{\text {app }}$ are experimental and approximation stress values, respectively.

In the optimization process, all hardening parameters are randomly distorted up to $20 \%$ and for each set of $b, Q, c, \gamma$ parameters, the error norm (15) is computed. The minimum error $(B=12.65 \%)$ is reached for the 


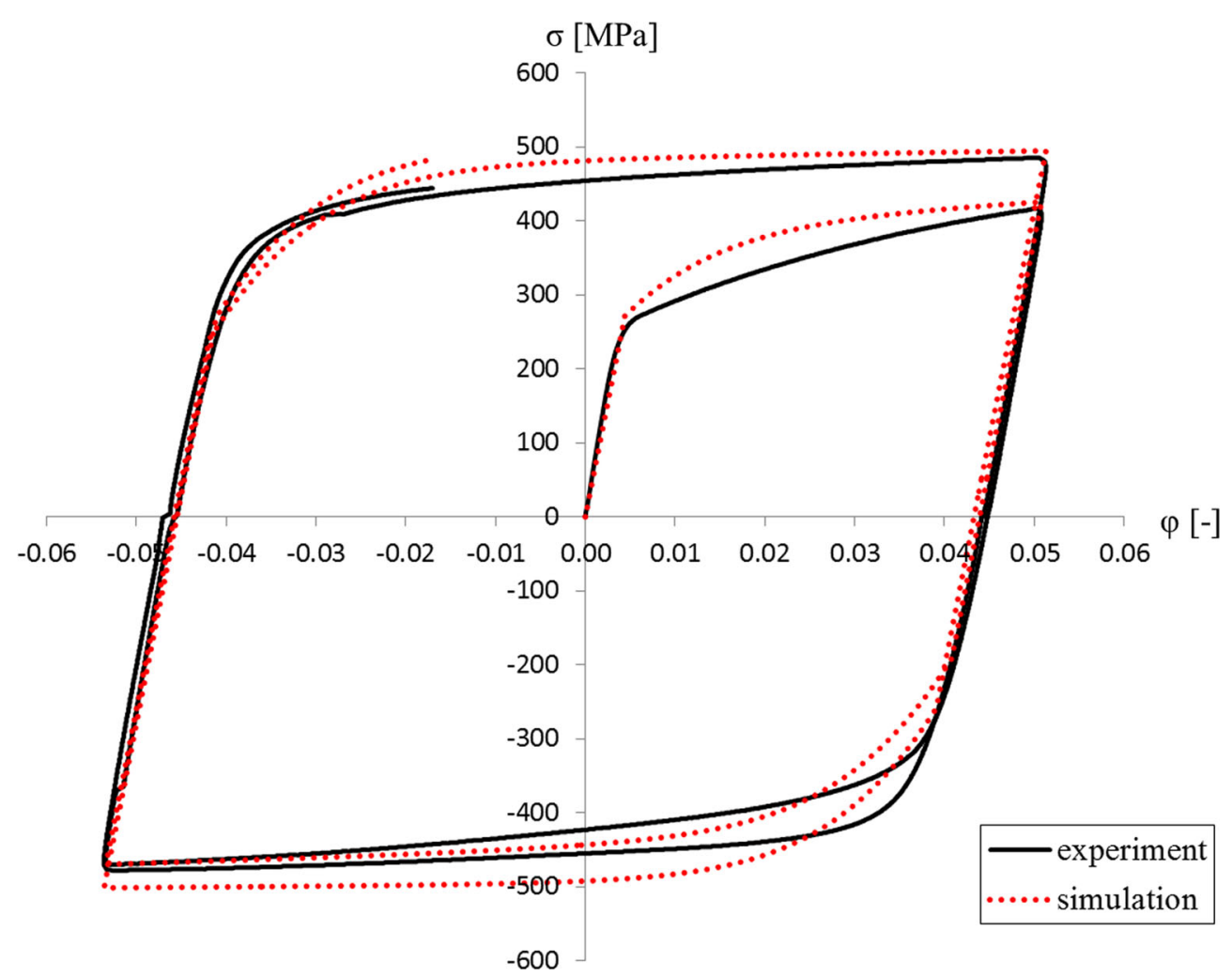

Fig. 6 Comparison of experimental and numerical curves after the optimization of hardening parameters

following data: $Q=138.42 \mathrm{MPa}, b=7.82, c=9617.90 \mathrm{MPa}, \gamma=118.91$. The comparison of numerical and experimental hysteresis loops is shown in Fig. 6.

It can be clearly seen in Fig. 6 that very good fitting of both curves is reached, even for only four hardening parameters taken into consideration. Better fitting requires using more complex hardening models, such as Chaboche and Lemaitre model.

\section{The application of fuzzy logic in enhancement of hardening parameters}

The optimization procedure mentioned in the previous section provides a very good agreement between cyclic tension-compression experiment and its numerical simulation. However, similar (small) error norms (16) can be obtained for various sets of hardening parameters. The best fit can be applied for the first loop and for the last loop, or a mild fit can be applied to all loops simultaneously without the preference of the particular one. In all these cases, the integral will be similar. Therefore, the question arises, which set provides the best simulation of the material response to the external load.

Fuzzy logic analysis is the tool which can help to select the best set of material hardening parameters assuming some uncertainty of the hardening data. In this research, the hardening parameters for combined hardening model $(Q, b, c, \gamma)$ are assumed to be fuzzy input variables. These parameters are randomly distorted up to $20 \%$. The triangular membership functions for each set of the input parameters are built. After that, the fuzzy analysis is performed. The fuzzy output variable $z \equiv B$ and its membership function $\mu(z)$ are calculated. The most reliable $z_{0}$ value (crisp value) is found in the defuzzification process. It is assumed that the best set of material hardening parameters provides the minimum of discrete $z_{0}$ value.

This procedure is time-consuming and may take several hours of computation depending on the number of the data randomizations, number of $\alpha$-levels and number of integration steps for a single hysteresis loop.

As the result of the fuzzy logic analysis, the following hardening parameters are computed: $Q=$ 134.31 MPa, $b=5.98, c=9925.36 \mathrm{MPa}, \gamma=101.22$. The approximation error $B$ calculated by Eq. 16 is $27.96 \%$. The comparison of numerical and experimental hysteresis loops is shown in Fig. 7. 


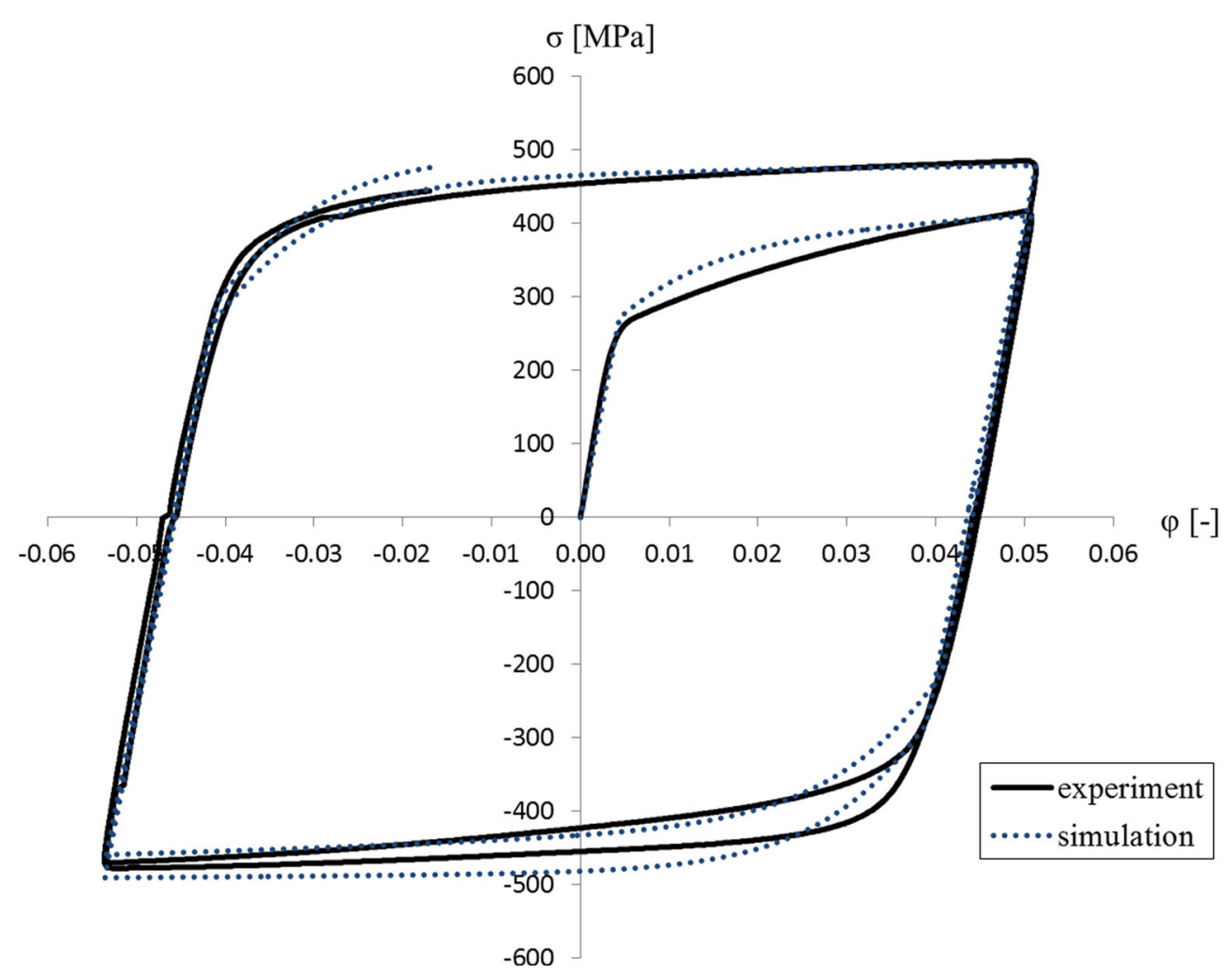

Fig. 7 Comparison of experimental and numerical curves after the fuzzy logic analysis

The advantage of using fuzzy logic over classical methods of statistics is that the fuzzy set theory considers the randomness of both input and output data. The nonlinear behavior of the mapping procedure is also included in the analysis.

\section{Summary and conclusions}

The proper selection of hardening parameters is necessary both for experimental and numerical calculations. In this paper, the procedure for the selection of hardening parameters of mixed nonlinear kinematic and isotropic hardening model with the use of authorial algorithm was implemented. The experimental as well as numerical hysteresis loops, for which hardening parameters were selected, were compared with the use of least-squares method. The analysis of results showed a relatively good fitting of both curves.

In order to improve the results of numerical simulation, the fuzzy set theory was applied. Fuzzy logic is a non-deterministic method for the analysis of data which is imprecisely defined. The application of fuzzy logic in plasticity theory is associated with the fact that many parameters are usually imprecise or uncertain. An example of this would be load magnitudes, material data and the friction coefficient which might change from one product to another. Therefore, the application of fuzzy logic for solving engineering problems for which input parameters are imprecisely defined is deliberate.

Nonlinear hardening models are good examples of fuzziness. The hardening parameters might be determined in an experimental test and optimized with the use of a numerical procedure. Better results might be achieved by the execution of the fuzzy analysis. In this research, the hardening parameters of mixed nonlinear kinematic and isotropic models were selected with the use of fuzzy logic. The variation of hardening parameters was assumed, and the fuzzification was done. As a mapping model, a system of differential equation associated with plasticity theory was used. Many numerical simulations were carried out, and the lowest discrete value obtained in a defuzzification step was selected as a final result.

The simulation shows the potential of application of fuzzy logic analysis for the determination of hardening parameters. The fuzzy set theory allows finding the variation of elastic-plastic material response when 
uncertainness of input parameters is taken into consideration. Therefore, the fuzzy analysis might be a useful tool in the analysis of metal forming processes.

Acknowledgements The authors would like to thank the anonymous reviewers for their constructive comments.

Open Access This article is distributed under the terms of the Creative Commons Attribution 4.0 International License (http:// creativecommons.org/licenses/by/4.0/), which permits unrestricted use, distribution, and reproduction in any medium, provided you give appropriate credit to the original author(s) and the source, provide a link to the Creative Commons license, and indicate if changes were made.

\section{References}

1. Ryu, H.-W., Kim, H.-T., Kim, Y.-J., Kim, J.-W.: Determination of combined hardening parameters to simulate deformation behavior of C(T) specimen under cyclic loading. Procedia Struct. Integr. 13, 1932-1939 (2018)

2. Jenitha, G., Saravanan, M., Vishnuvardhan, S., Raghava, G., Babu, V.N.: Evaluation of cyclic hardening parameters for type 304LN stainless steel. Appl. Mech. Mater. 592-594, 1200-1204 (2014)

3. Kim, J.W., Choi, M.R., Kim, Y.J.: Effect of loading rate on the fracture behavior of nuclear piping materials under cyclic loading conditions. NET 48(6), 1376-1386 (2016)

4. Kim, J.W., Choi, M.R., Kim, Y.J.: Fracture Behavior of Aged CF8A Austenite Cast Stainless Steel Under Dynamic and Cyclic Loading Conditions. In: Proceedings of the ASME 2017 Pressure Vessels and Piping Conference (PVP2017), pp. V03BT03A007. American Society of Mechanical Engineerings (2017)

5. Yang, M., Pan, Y., Yuan, F., Zhu, Y., Wu, X.: Back stresses strengthening and strain hardening in gradient structure. Mater. Res. Lett. 4(3), 145-151 (2016)

6. Silvestre, E., Mendiguren, J., Galdos, L., de Argandofia, E.: Influence the number of tensile/compression cycles on the fitting of a mixed hardening model: roll levelling process case study. Key Eng. Mater. 554-557, 2375-2387 (2013)

7. Koo, S., Han, J., Marimuthu, K.P., Lee, H.: Determination of Chaboche combined hardening parameters with dual backstress for ratcheting evaluation of AISI 52100 bearing steel. Int. J. Fatigue 122, 152-163 (2019)

8. Halama, R., Sofer, M., Fojtík, F.: Choice and calibration of cyclic plasticity model with regard to subsequent fatigue analysis. Eng. Mech. 19(2/3), 87-97 (2012)

9. Abdel-Karim, M.: Modified kinematic hardening rules for simulation of ratcheting. Int. J. Plast. 25(8), $1560-1587$ (2009)

10. Ho, K.: Effect of the rate dependence of nonlinear kinematic hardening rule on relaxation behaviour. Int. J. Solid Struct. 45(3-4), 821-839 (2008)

11. Robert, L., Velay, V., Decultot, N., Ramde, S.: Identification of hardening parameters using finite element models and full-field measurement: some case studies. J. Strain Anal. Eng. 47(1), 3-17 (2012)

12. Notta-Cuvier, D., Langrand, B., Markiewicz, E., Lauro, F., Portemont, G.: Identification of Johnson-Cook's viscoplastic model parameters using the virtual fields method: application to titanium alloy Ti6Al4V. Strain 49, $22-45$ (2013)

13. Placidi, L., Barchiesi, E., Della Corte, A.: Identification of two-dimensional pantographic structures with a linear D4 orthotropic second gradient elastic model accounting for external bulk double forces. In: dell'Isola, F., Sofonea, M., Steigmann, D. (eds.) Mathematical Modelling in Solid Mechanics, pp. 211-232. Springer, Berlin (2017)

14. Misra, A., Poorsolhjouy, P.: Identification of higher-order elastic constants for grain assemblies base upon granular micromechanics. MEMOCS 3(3), 285-308 (2015)

15. Giorgio, I., Scerrato, D.: Multi-scale concrete model with rate dependent international friction. Eur. J. Environ. Civ. Eng. 21(7-8), 821-839 (2017)

16. De Angelo, M., Spagnuolo, M., D’Annibale, F., Pfaff, A., Hoschke, K., Misra, A., Dupuy, C., Peyre, P., Dirrenberger, J., Pawlikowski, M.: The macroscopic behavior of pantographic sheets depends mainly on their microstructure: experimental evidence and qualitative analysis of damage in metallic specimens. Continuum Mech. Thermodyn. (2019). https://doi.org/ 10.1007/s00161-019-00757-3

17. Möller, B., Beer, M.: Fuzzy Randomness. Uncertainty in Civil Engineering and Computational Mechanics. Springer-Verlag, Berlin (2004)

18. Skrzat, A., Wójcik, M.: The application of fuzzy logic in engineering applications. RUTMech 35(90/4), 505-518 (2018)

19. Skrzat, A.: Fuzzy logic application to strain-stress analysis in selected elastic-plastic material model. Arch. Metall. Mater. 56(2), 559-568 (2011)

20. Skrzat, A., Wójcik, M., Bạk, Ł.: Określenie parametrów umocnienia materiału w próbie Heyera z zastosowaniem metod logiki rozmytej. In: Śliwa, R., Pawłowska, B. (eds.) Proceedings of XI Konferencja Naukowa Zintegrowane studia podstaw deformacji plastycznej metali, p. 143. Rzeszow University of Technology Publishing House, (2018)

21. Ozben, T., Huseyinoglu, M., Arsian, N.: Fuzzy logic model for the prediction failure analysis of composite plates under various cure temperatures. J. Braz. Soc. Mech. Sci. Eng. 36(3), 443-448 (2014)

22. Akkurt, S., Tayfur, G., Can, S.: Fuzzy logic model for the prediction of cement compressive strength. Cem. Concr. Res. 34, 1429-1433 (2004)

23. Vygnanov, A.A., Fironov, A.N.: Fuzzy-logic expert system to estimate the risk of high-speed railways projects. Autobusy 12, 651-655 (2017)

24. Guclu, R., Metin, M.: Fuzzy logic control of vibrations of a light rail transport vehicle in use in Instanbul traffic. J. Vib. Control 15(9), 1423-1440 (2009)

25. Sasmal, S., Ramanjaneyulu, K., Iyer, N.R.: Condition ranking and rating of bridges using fuzzy logic. In: Dadios, E. (ed.) Fuzzy Logic_Emerging Technologies and Applications, pp. 175-202. IntechOpen, Arizona (2012)

26. Dunne, F., Petrinic, N.: Introduction to Computational Plasticity. Oxford University Press, New York (2005) 
27. Gurtin, M.E., Reddy, B.D.: Alternative formulations of isotropic hardening for Mises materials, and associated variational inequalities. Continuum Mech. Thermodyn. 21, 237-250 (2009)

28. Tsakmakis, Ch.: Kinematic hardening rules in finite plasticity. Part I: a constitutive approach. Continuum Mech. Thermodyn 8, 215-231 (1996)

29. Tsakmakis, Ch.: Kinematic hardening rules in finite plasticity. Part II: some examples. Continuum Mech. Thermodyn. 8, 233-246 (1996)

30. Toros, S.: Parameters determination of Yoshida Uemori model through optimization process of cyclic tension-compression test and V-bending springback. Lat. Am. J. Solids Struct. 13, 1893-1911 (2016)

31. Aziz M.Z.B.A.: Identification of kinematic hardening parameters for mild steel by cyclic loading. Universiti Malaysia Pahang. http://umpir.ump.edu.my/id/eprint/8630/1/CD8056_@_57.pdf (2013). Accessed 13 March 2019

32. Seisenbacher, B., Winter, G., Grün, F.: Improved approach to determine the material parameters for a combined hardening model. MSA 9, 357-367 (2018)

33. Harris, J.: Fuzzy Logic Applications in Engineering Science. Springer, Dordreht (2006)

34. Hossain, M.A., Shill, P.C., Saker, B., Murase, K.: Optimal fuzzy model construction with statistical information using genetic algorithm. IJCSIT 3(6), 241-257 (2011)

35. Seibert, T., Lehn, J., Schwan, S., Kollmann, F.G.: Identification of material parameters for inelastic constitutive models: stochastic simulations for the analysis of deviations. Continuum Mech. Thermodyn. 12(2), 95-120 (2000)

36. Sobol, I.M., Tarantola, S., Gatelli, D., Kucherenko, S.S., Mauntz, W.: Estimating the approximation error when fixing unessential factors in global sensitivity analysis. Reliab. Eng. Syst. Safe. 92(7), 957-960 (2007)

Publisher's Note Springer Nature remains neutral with regard to jurisdictional claims in published maps and institutional affiliations. 